\title{
Surfactant-induced gradients in the three-dimensional Belousov-Zhabotinsky reaction
}

\author{
Dennis Kupitz, ${ }^{1}$ Sergio Alonso, ${ }^{2}$ Markus Bär, ${ }^{2}$ and Marcus J. B. Hauser ${ }^{1, *}$ \\ ${ }^{1}$ Institut für Experimentelle Physik, Abteilung Biophysik, Otto-von-Guericke Universität Magdeburg, Universitätsplatz 2, \\ 39106 Magdeburg, Germany \\ ${ }^{2}$ Physikalisch-Technische Bundesanstalt, Abbestraße 2-12, 10587 Berlin, Germany
}

(Received 9 August 2011; published 10 November 2011)

\begin{abstract}
Scroll waves are prominent patterns formed in three-dimensional excitable media, and they are frequently considered highly relevant for some types of cardiac arrhythmias. Experimentally, scroll wave dynamics is often studied by optical tomography in the Belousov-Zhabotinsky reaction, which produces $\mathrm{CO}_{2}$ as an undesired product. Addition of small concentrations of a surfactant to the reaction medium is a popular method to suppress or retard $\mathrm{CO}_{2}$ bubble formation. We show that in closed reactors even these low concentrations of surfactants are sufficient to generate vertical gradients of excitability which are due to gradients in $\mathrm{CO}_{2}$ concentration. In reactors open to the atmosphere such gradients can be avoided. The gradients induce a twist on vertically oriented scroll waves, while a twist is absent in scroll waves in a gradient-free medium. The effects of the $\mathrm{CO}_{2}$ gradients are reproduced by a numerical study, where we extend the Oregonator model to account for the production of $\mathrm{CO}_{2}$ and for its advection against the direction of gravity. The numerical simulations confirm the role of solubilized $\mathrm{CO}_{2}$ as the source of the vertical gradient of excitability in reactors closed to the atmosphere.
\end{abstract}

DOI: 10.1103/PhysRevE.84.056210 PACS number(s): 05.45.-a, 05.65.+b, 82.40.Ck, 89.75.Kd

\section{INTRODUCTION}

Excitable media play an important role in many biological systems, since they support signal propagation in an intra- and extracellular context $[1,2]$. While in two-dimensional excitable systems the typical structures are rotating spiral waves, this role is played by scroll waves in three-dimensional systems. A scroll wave can be considered as a three-dimensional (3D) extension of a spiral wave.

Cardiac tissue is an example of three-dimensional excitable media where scroll waves are believed to be at the basis of some types of cardiac arrhythmias, like ventricular tachycardia [3]. In the ventricle a scroll wave of electrical activity acts as an autonomous pacemaker that takes over the control over the dynamics of the ventricle from the natural pacemaker, the sine node. Due to the importance of such a situation, numerous simulation studies of scroll wave dynamics in the context of cardiac arrhythmias have been performed [4].

Since it is very difficult to visualize excitable waves in cardiac tissue in three dimensions, experimental studies are often performed using a chemical model system, namely the Belousov-Zhabotinsky (BZ) reaction [5-7]. Here, threedimensional patterns of excitability can be made visible by optical tomography, which was introduced by Winfree and coworkers [8] and has since been used in a series of investigations [9-12].

The shape of the filament of a scroll wave, i.e., the line connecting all singular points of the scroll wave, is prone to be destabilized by several instabilities. Straight scroll waves with linear filaments may be subjected to three types of instabilities causing the filaments to adopt different shapes. For instance, when the scroll wave is subjected to the negative line tension instability, the filament starts to grow in extension and loses its originally straight shape $[10,13,14]$. Eventually, this type

*marcus.hauser@ovgu.de of instability may lead to chaotic scroll patterns, the so-called scroll wave turbulence or Winfree turbulence [15].

Scroll waves may also lose their stability by a form of threedimensional meandering. In systems with anchored scroll waves, which correspond to systems with nonflux boundary conditions, this instability causes a certain elongation of the filament, which adopts a zig-zag shape [16,17]. Recently, this scenario has been also observed experimentally [10].

Finally, scroll waves may also suffer the so-called sproing instability [18]. This instability results from a phase shift along the filament, which corresponds to a twist along the filament. When the twist is sufficiently large, the filament bends and eventually collides and attaches to the boundary of the medium (e.g., the reactor wall) $[9,19,20]$. Theoretical studies have shown that a gradient in excitability oriented parallel to the filament induces a twist in the scroll wave, since the higher rotation frequency at the more excitable end of the scroll wave needs to be compensated [21,22]. Experimental realizations of twisted scroll waves were studied using gradients of temperature [20], illumination intensity [23], and concentration of oxygen [9]. A theory of the scroll instability stemming from gradients in excitability and a related numerical stability analysis have been given in Ref. [22]. External gradients of excitability have also been studied in scroll rings, where gradients of temperature [24] and electric current $[25,26]$ may cause the reorientation of the scroll ring with respect to the direction of the gradient, as well as scroll ring shrinkage or growth.

In the classical version of the $\mathrm{BZ}$ reaction malonic acid is brominated in a strongly acidic medium, producing $\mathrm{CO}_{2}$, which leads to the formation of $\mathrm{CO}_{2}$ gas bubbles in the reaction medium. The gas bubbles are an undesired feature that very often disturbs or even destroys the pattern under investigation. Therefore, methods have been developed to suppress, diminish, or retard the formation of such gas bubbles. One possibility is to replace malonic acid by 1,4-cyclohexanedione, which does not form $\mathrm{CO}_{2}$ during the $\mathrm{BZ}$ reaction [27,28]. In 
the classical BZ system, a popular technique consists in the addition of small amounts of the surfactant sodium dodecyl sulfate (SDS) to the BZ reaction medium [9,29-33]. It is generally assumed that, when added at concentrations below its critical micelle concentration (CMC), SDS does not affect the dynamics of the BZ system [29,31].

The general picture changes when the $\mathrm{BZ}$ reaction is run in compartmented media. Such conditions are obtained when a surfactant or phospholipid is added to the BZ medium at a concentration that exceeds its critical micelle (or vesicle) concentration. Typical features of the oscillatory BZ reaction, like the periods and amplitudes of oscillations and the lengths of the induction period, are affected by micelles and vesicles in the reaction medium [34-37]. Surfactants and phospholipid bilayers also exert an influence on the spatiotemporal patterns formed in the BZ system [38,39]. For instance, the velocity of reaction waves decreases as the SDS content is augmented and the viscosity of the medium is increased [39]. In phospholipid lamellar phases, the BZ reaction interacts with the lamellar structure of the phospholipid arrangement, leading to a mutual interplay between the reaction and the phospholipid matrix [40].

In the present article, we study the effect of addition of sub-CMC concentrations of SDS on the dynamics of BZ scroll waves. While the addition of SDS suppresses or delays $\mathrm{CO}_{2}$ bubble formation, it may lead to the generation of vertical gradients of $\mathrm{CO}_{2}$ in the extended three-dimensional reaction system. The effect of such $\mathrm{CO}_{2}$-induced gradients of excitability on the dynamic behavior of the scroll waves will be studied. In addition, we also show how such gradients may be avoided.

The paper is organized as follows: In the next section we describe the experimental materials and methods, and we present the experimental results in Sec. III. Here the dynamics of the scroll waves obtained in reactors that are either open or closed to the atmosphere are described. The differences in the dynamics are attributed to the fate and the accumulation of the reaction product $\mathrm{CO}_{2}$ in the reactor. This hypothesis is at the basis of the model, which is introduced and described in Sec. IV. The results of the numerical simulations are presented in Sec. V, which is followed by the discussion (Sec. VI), where we interpret and compare our experimental and numerical findings. Last, we close the paper with our conclusions (Sec. VII).

\section{EXPERIMENTAL METHODS}

\section{A. Chemical preparation}

Experiments were carried out using the ferroin-catalyzed $\mathrm{BZ}$ reaction embedded in an $0.1 \%$ (weight by volume) agarose (type VII, Sigma) gel matrix in order to prevent any hydrodynamic effects perturbing the wave fronts. The gel was stiff enough to avoid blurring of the edges of the scroll wave due to vibrations induced by the stepper motor of the tomographic apparatus. To reduce the production of $\mathrm{CO}_{2}$ bubbles, the surfactant SDS was added at low concentrations.

For preparation of $10 \mathrm{ml}$ of the aqueous solution the agarose was heated until it became clear and without streaks (at $90^{\circ} \mathrm{C}$ ). After the solution had cooled to $55^{\circ} \mathrm{C}$, the water lost by evaporation was compensated. The components of the BZ reaction (except the catalyst, i.e., ferroin) and the SDS were added when the temperature had reached $36^{\circ} \mathrm{C}$. Ferroin was added at $T=27^{\circ} \mathrm{C}$, just before the solution was poured into the cylindrical cuvette. The initial concentrations were $50 \mathrm{mM}$ malonic acid (Merck), $50 \mathrm{mM}$ sodium bromate (Merck), 180 or $250 \mathrm{mM}$ sulfuric acid (Fluka) and $0.5 \mathrm{mM}$ ferroin. The initial concentration of SDS lay in the range of 0.05 to $0.40 \mathrm{mM}$; however most of the experiments were run at $0.20 \mathrm{mM}$ SDS. The reaction mixture was allowed to cool down to $21 \pm 1{ }^{\circ} \mathrm{C}$; i.e., the temperature controlled by the air conditioning system of the laboratory and the experiments were performed at this temperature.

Under our reaction conditions the CMC of SDS was found to lie at $0.25 \mathrm{mM}$, as seen from UV-visible spectra of ferroin in acidic SDS solutions. Once SDS micelles are formed, ferroin is sequestered into the micelles, as seen from the spectra. The measured value of the CMC is in good agreement with the fact that the CMC is known to decrease with increasing ionic strength [37].

In two-dimensional (2D) systems the recipes with 180 and $250 \mathrm{mM}$ sulfuric acid yield meandering and rigidly rotating spiral waves, respectively. The same types of trajectories are expected for the filaments of the three-dimensional scroll waves.

\section{B. Initiation of scroll waves}

Scroll waves were initiated by the partition method [9,10,19]. $8.7 \mathrm{ml}$ of the reaction solution were filled into the reaction cuvette made of fluorinated polyethylene (FEP, inner diameter $21 \mathrm{~mm}$, height $100 \mathrm{~mm}$, height of the gel matrix $25 \mathrm{~mm}$ ). The reaction volume was divided into two compartments by a polyethylene terephthalate (PET) sheet of $\sim 0.1$-mm thickness. A cylindrical wave was initiated by a silver wire (diameter $0.5 \mathrm{~mm}$ ) in the immediate vicinity of the PET sheet and close to the wall of the cuvette. When the wave front hit the partitioning sheet, a segment of the cylindrical wave persisted, forming two contact lines with this sheet. These lines then moved away from the ignition site, one toward the center and the other toward the wall of the cuvette. When the latter anchored at the cuvette wall, the partitioning sheet was carefully removed, and the gel closed the gap formed by the removal of the partitioning sheet. The first, inwardly moving reaction front was left as an open wave edge in the bulk of the reaction volume and curled in to form a scroll wave.

\section{Tomography and data reconstruction}

The reaction cuvette was monitored in the tomographic setup $[9,10]$, the illumination of which was provided by a blue LED $(\lambda \sim 470 \mathrm{~nm}$, bandwidth $50 \mathrm{~nm})$. One tomographic sample consists of 100 projections through the reaction cuvette taken at angles $1.8^{\circ}$ apart from each other. The spatial

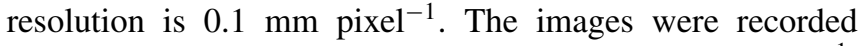
by a CCD camera (Hamamatsu C3077) at 25 frames $\mathrm{s}^{-1}$, digitized, and stored on a computer for later analysis and image reconstruction.

Parallel optics is a necessary requirement for the tomographic detection. Therefore the cylindrical reaction cuvette 
was immersed into a square-shaped cuvette for index matching. The refractive indices of the reaction medium, the FEP cuvette, and the index matching liquid $\left(230 \mathrm{mM} \mathrm{K}_{2} \mathrm{SO}_{4}\right)$ were $1.339,1.338$, and 1.339 , respectively.

The recorded samples of the reaction volume are, slicewise, the Radon transform of the ferroin and ferriin concentration [41]. Thus, the entire information about the spatial concentration distribution is contained within the tomographic samples (projections). The three-dimensional structure has to be reconstructed from all projections (i.e., the tomographic raw data) by inverting the Radon transform, as shown in Refs. [9,10]. The result of this operation consists in a stack of slicewise reconstructed concentration data, from which isoconcentration surfaces are calculated.

\section{Determination of the filament and its phase}

The filament of a scroll wave is obtained from the reconstructed 2D intersections through the scroll wave. Each of these 2D slices shows a spiral wave. To extract the position of the spiral tip, a series of successive images is superimposed onto each other, until the spiral wave has completed a revolution. The area never visited by the spiral tip is the spiral core. The edges of the spiral core are detected by a Sobel filter. The $x$ and $y$ coordinates of the center of mass of the spiral core are determined as the position of the filament. This procedure is repeated for all slices intersecting the scroll wave (usually 250 slices at a distance of $0.1 \mathrm{~mm}$ ), defining the filament of the scroll wave.

The phase along a filament is determined from the stack of horizontal slices of the reconstructed data. Each slice represents an intersection of the scroll wave with the horizontal surfaces. To determine the phase, a unit circle with a coordinate system from 0 to $2 \pi$ is placed around the spiral wave of each reconstructed $2 \mathrm{D}$ slice (Fig. 1), such that the center of the unit circle is located at the spiral tip, forming the origin of the coordinate system. Next, a line is drawn that connects the origin to the point where the wave front intersects the unit circle. The phase angle between this line and the coordinate system is measured. The error for the slice offset is estimated at $\pm 5^{\circ}$. The spiral tip is ideally suited as a reference point and can be determined in every slice of the reconstructed data.

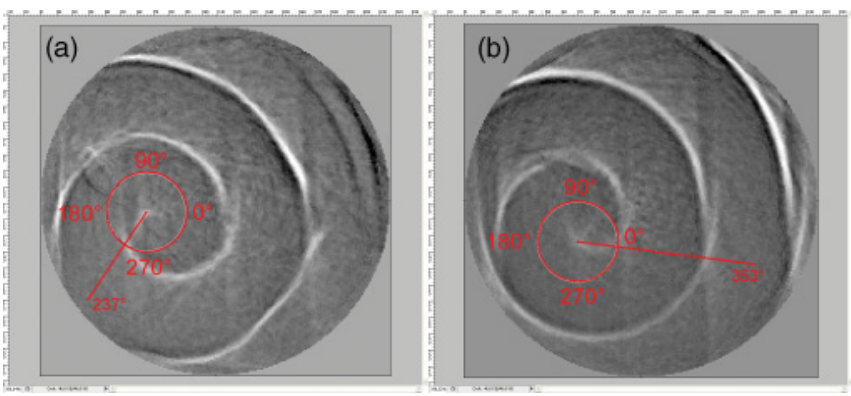

FIG. 1. (Color online) Two reconstructed slices of a scroll wave at $315 \mathrm{~min}$ after initiation of the experiment. The phase of the spiral wave is (a) $237^{\circ}$ in the topmost slice of the sample and (b) $353^{\circ}$ in the slice $3.0 \mathrm{~mm}$ below the top of the sample. Sample diameter is $20 \mathrm{~mm}$.

\section{EXPERIMENTAL RESULTS}

\section{A. Effect of SDS concentration on scroll waves}

SDS lowers the interfacial tension between the BZ liquid and $\mathrm{CO}_{2}$ (micro-) bubbles, thus providing for an effective solubilization of $\mathrm{CO}_{2}$ in the gel-entrapped medium. Therefore, the SDS concentration is expected to have an influence on the formation of scroll waves and on their tomographic observation. We performed experiments where the SDS concentration was varied from 0.00 to $0.40 \mathrm{mM}$. In these experiments the reactor was sealed with a teflon cover once a scroll wave in the cylindrical reactor was initiated.

A pronounced effect of the SDS concentration on the formation and detectability of the scroll wave is observed (Fig. 2). At low concentrations of SDS, e.g., at 0.05 or $0.10 \mathrm{mM}$ SDS, a considerable amount of macroscopic $\mathrm{CO}_{2}$ bubbles is still produced, which cannot dissolve due to the high interfacial tension between the $\mathrm{CO}_{2}$ bubbles and the $\mathrm{BZ}$ medium. Although not always simple to detect, the scroll wave remains straight throughout the entire experiment. As seen from the rough, punctured, and partly discontinuous isoconcentration surface in Fig. 2(a), the reconstruction of the scroll waves at SDS concentrations as low as 0.05 or $0.10 \mathrm{mM}$ is compromised by the formation of $\mathrm{CO}_{2}$ bubbles.

An increase of the SDS content in the reaction mixture suppresses the formation of macroscopic gas bubbles. At a SDS content of $0.20 \mathrm{mM}$, i.e., slightly below the CMC, the scroll wave can easily be reconstructed, and tomographic artifacts arising from $\mathrm{CO}_{2}$ bubbles are rare [Fig. 2(b)]. A further increase in SDS concentration, however, drives the reaction system past the CMC. Once this is reached, ferroin is entrapped into the micelles, thus leading to a loss of contrast and to light scattering. Ultimately, for $\mathrm{SDS}=0.40 \mathrm{mM}$, the reaction conditions are those of a partitioned medium [Fig. 2(c)] [39].

The surfactant SDS lowers the interfacial tension between the BZ liquid and the $\mathrm{CO}_{2}$ bubbles. In the presence of SDS concentrations around $0.20 \mathrm{mM}$ only nanoscopically small $\mathrm{CO}_{2}$ bubbles are formed and in equilibrium with the BZ
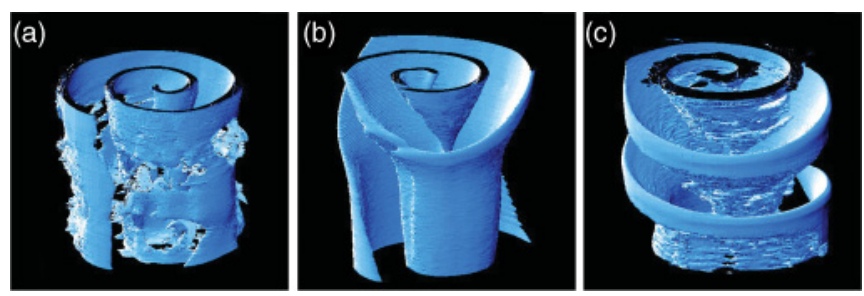

FIG. 2. (Color online) Isoconcentration surfaces of three scroll waves at $t=200 \mathrm{~min}$ after initiation with three different SDS concentrations: (a) $0.10 \mathrm{mM}$ SDS, (b) $0.20 \mathrm{mM}$ SDS, and (c) $0.40 \mathrm{mM}$ SDS. Note that the strength of twisting increases with the SDS concentration in the medium. The quality of the reconstructed tomographic images depends on the SDS content: while (a) at low SDS contents still a substantial amount of $\mathrm{CO}_{2}$ bubbles are formed, leading to a rough and punctured isoconcentration surface, (b) SDS concentrations at $\sim 0.20 \mathrm{mM}$ lead to smooth and continuous reconstructions; for (c) SDS concentrations above the CMC the reconstructed images become rougher again. Sample dimensions: $\sim 22 \times 22 \times 22 \mathrm{~mm}^{3}$. 
medium. In fact, such a situation does not compromise the parallelism of the light beams, thus enabling the tomographic observation of the reaction dynamics [Fig. 2(b)]. However, the nanoscopic $\mathrm{CO}_{2}$ bubbles have a lower density than the surrounding medium. Consequently, in the gravity field, they are slowly transported to the surface of the reactor and eventually transferred into the gas phase.

In a closed reactor $\mathrm{CO}_{2}$ cannot escape to the atmosphere and it tends to accumulate underneath the lid of the reactor. Through the equilibrium

$$
\mathrm{CO}_{2}+\mathrm{H}_{2} \mathrm{O} \rightleftharpoons \mathrm{HCO}_{3}^{-}+\mathrm{H}^{+},
$$

the $\mathrm{CO}_{2}$ gradient induces a gradient in proton concentration. Since the excitability of the BZ medium increases with the $\mathrm{H}^{+}$ concentration $[5,42]$, this leads to a gradient of excitability. This vertical gradient has a parallel orientation to the filament of the scroll wave, and such gradients are known to induce a twist of the scroll wave [9,23,24].

The SDS concentration also plays a role in the kinetics of the twist formation. In experiments with SDS concentrations above $0.05 \mathrm{mM}$ in closed reactors the scroll wave develops a twist within a few hours. Figures 2 and 3 show that the twist starts at the top of the reaction medium and propagates downward in the reactor. The twist rate increases with the SDS concentration in the $\mathrm{BZ}$ medium, leading to scroll waves with a larger number of twists in media with higher SDS content. For instance, at $t=200 \mathrm{~min}$ the scroll waves are twisted by $\sim 2 / 3 \pi, 2.0 \pi$, and $3.5 \pi$ for BZ reactions containing $0.10,0.20$, and $0.40 \mathrm{mM}$ SDS, respectively (Figs. 2 and 3). Furthermore, for a given instant, where the twist has not yet reached from the top to the bottom of the reactor, the extension of the twist along the $z$ direction also augments slightly with the SDS content (Fig. 3). The SDS concentration-dependent twist rates, twist extensions, number of twists, and the total dephasing of the filaments are compiled in Table I.

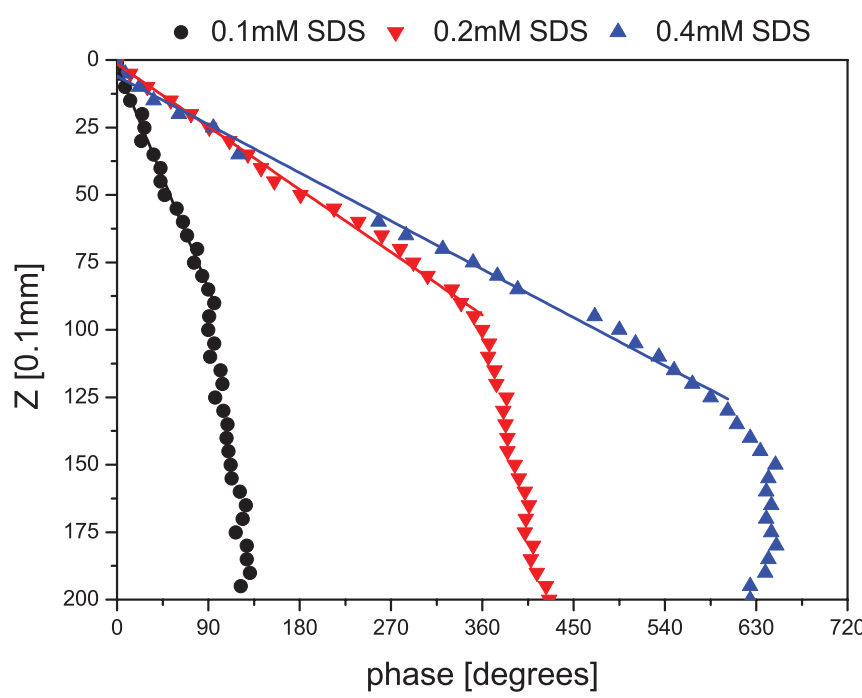

FIG. 3. (Color online) Twisting of the three different scroll waves against the height of the BZ medium 200 min after initiation of the experiment.
TABLE I. Data of the twists at three different SDS concentrations. All data were measured 200 min after the initiation of the scroll wave.

\begin{tabular}{lccc}
\hline \hline Twist of scroll wave & $0.1 \mathrm{mM}$ SDS & $0.2 \mathrm{mM}$ SDS & $0.4 \mathrm{mM} \mathrm{SDS}$ \\
\hline Twist rate $\left({ }^{\circ} \mathrm{mm}^{-1}\right)$ & 11.0 & 38.5 & 50.0 \\
Twist extension $(\mathrm{mm})$ & 9.0 & 9.5 & 13.0 \\
Number of twists & 0.3 & 1.0 & 1.8 \\
Phase change & $2 / 3 \pi$ & $2 \pi$ & $3.5 \pi$ \\
\hline
\end{tabular}

\section{B. Dynamics of rigidly rotating scroll waves}

Scroll waves with a straight, rigidly rotating filament were initiated in a BZ medium containing $0.20 \mathrm{mM}$ SDS and $250 \mathrm{mM}$ sulfuric acid, and their evolution was followed by tomography. The cylindrical reactor was either kept open to the atmosphere or closed with a teflon lid immediately after scroll wave initiation.

Interestingly, the scroll waves develop differently depending on whether the reactor is closed or open to the atmosphere. In a reactor open to the atmosphere, the originally straight scroll wave preserves its shape throughout the experiment, as observed from the reconstructed isoconcentration images [Fig. 4(b)]. In contrast, with time, an initially straight scroll wave in a closed reactor develops a twist. The twist starts at the top of the reaction medium, i.e., just below the covering lid of the reactor, and it propagates downward along the scroll wave [Fig. 4(a)]. In both cases, however, the filaments remain straight [Figs. $4(\mathrm{c})$ and $4(\mathrm{~d})$ ]. The only difference in
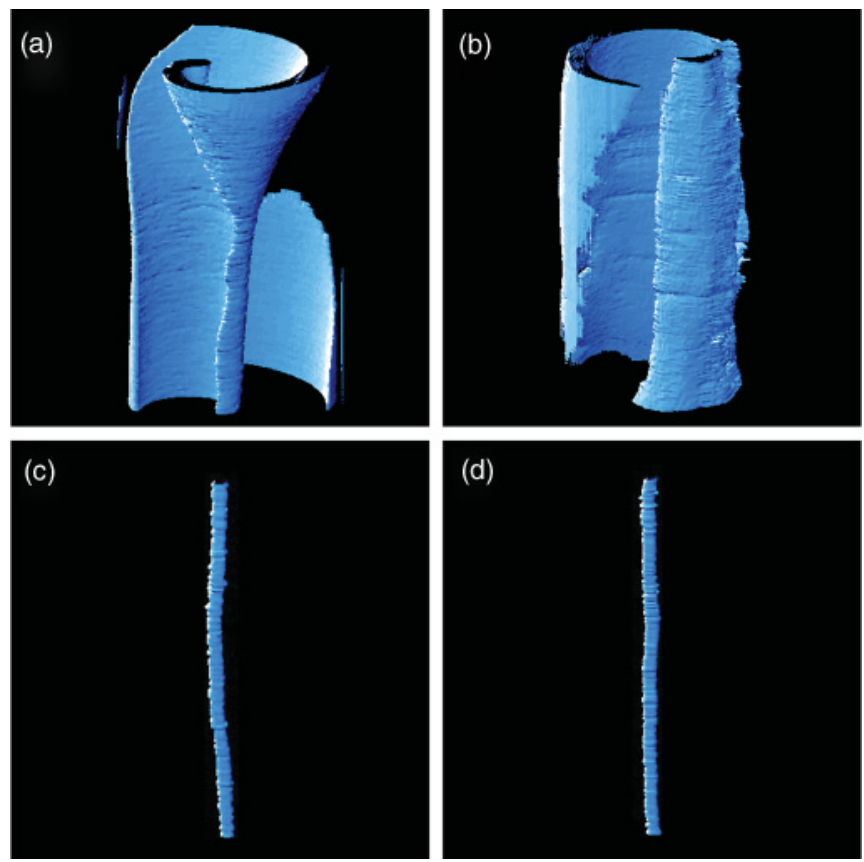

FIG. 4. (Color online) Isoconcentration surfaces of rigidly rotating scroll waves $200 \mathrm{~min}$ after initiation in a closed reactor and in a reactor open to the atmosphere. While (a) in a closed reactor the originally straight scroll wave develops a twist in its upper half, (b) a scroll wave remains straight in an open reaction container. In both cases the filament of the scroll wave remains straight (c) for a closed reactor and (d) in a reactor open to the atmosphere. Sample dimensions: $23.0 \times 23.0 \times 20.1 \mathrm{~mm}^{3}$. 
these experiments consists in the openness or closeness of the reactor to the atmosphere and hence in the ability of the reaction medium to release $\mathrm{CO}_{2}$ to the gas phase. While $\mathrm{CO}_{2}$ escapes effectively from an open reactor, this is impossible in a closed reactor; hence, $\mathrm{CO}_{2}$ accumulates at the top of such a reactor, forming a vertical gradient of $\mathrm{CO}_{2}$ concentration that corresponds to a vertical gradient in excitability. Since these gradients are oriented parallel to the filament, they induce a twist of the scroll wave. With time the twist expands downward into the BZ medium.

\section{Dynamics of meandering scroll waves}

Experiments similar to those described in the previous section have also been performed using meandering scroll waves. In these experiments the concentration of sulfuric acid was lowered to $180 \mathrm{mM}$, producing outward meandering spiral waves with four loops. Again, both the scroll wave and its filament were originally straight. The addition of $0.20 \mathrm{mM}$ SDS enables the formation of vertical gradients of $\mathrm{CO}_{2}$ concentration, which may either persist in a reactor closed to the atmosphere or vanish in a reactor open to the atmosphere. The meandering scroll in a reactor without a gradient parallel to the filament, as realized in an open reactor, remains straight [Fig. 5(b)]. Its filament also remains straight [Fig. 5(d)]. However, small perturbations of the filament may lead to a persistent zig-zag-shaped modulation of the filament. This corresponds to the transition between quasi-2D meandering
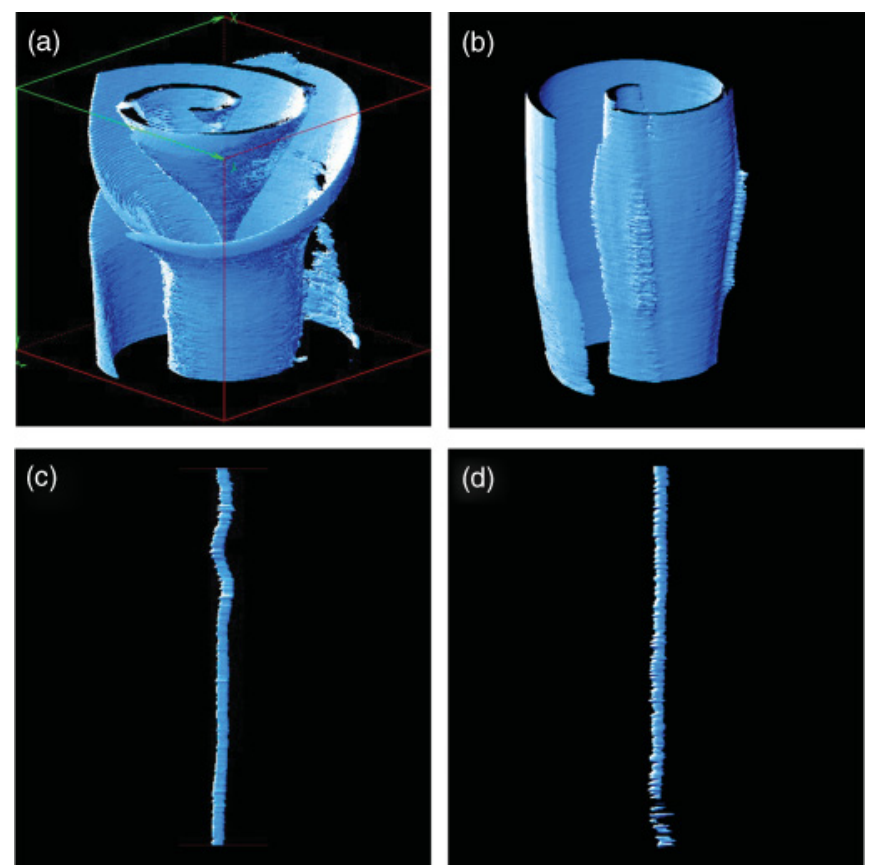

FIG. 5. (Color online) Isoconcentration surfaces of meandering scroll waves $200 \mathrm{~min}$ after initiation in a closed reactor and in a reactor open to the atmosphere. While (a) in a closed reactor the originally straight scroll wave develops a twist in it upper half, (b) a scroll wave remains straight in an open reaction container. In (c) a closed reactor the filament becomes zig-zag shaped. The deformation starts from the top of the reactor. (d) The filament remains straight in a reactor open to the atmosphere. Sample dimensions: $23.0 \times 23.0 \times 20.1 \mathrm{~mm}^{3}$.
TABLE II. Dynamics of scroll waves in the presence and absence of a gradient parallel to the direction of the filament.

\begin{tabular}{lllll}
\hline \hline Scroll wave & \multicolumn{2}{c}{ With gradient } & \multicolumn{2}{c}{ Without gradient } \\
\hline Wave type & Scroll & Filament & Scroll & Filament \\
Rigidly rotating & Twisted & Straight & Straight & Straight \\
Meandering & Twisted & Zig-zag & Straight & Zig-zag \\
\hline \hline
\end{tabular}

to $3 \mathrm{D}$ meandering of a filament, as described by Rusakov et al. [43].

By contrast, a meandering scroll wave in the presence of a vertical $\mathrm{CO}_{2}$ gradient starts to twist at the top of the reactor [Fig. 5(a)]. With time the twist propagates downward. The originally straight filament is subjected to a 3D-meandering instability and becomes zig-zag shaped [Fig. 5(c)]. Again, the deformation of the filament starts at the top of the reactor and propagates downward along the filament. The dynamic behavior of meandering and rigidly rotating scroll waves in the presence and absence of a vertical $\mathrm{CO}_{2}$ gradient is compiled in Table II.

\section{MODEL}

\section{A. The Oregonator model}

We consider the scaled and dimensionless Oregonator model [44] of the BZ reaction. It consists of two reactiondiffusion equations, one equation for the fast activator $u$ and the other for the slow inhibitor $v$ :

$$
\begin{aligned}
& \frac{\partial u}{\partial t}=\frac{1}{\epsilon}\left[u(1-u)-f v \frac{u-q}{u+q}\right]+D_{u} \nabla^{2} u, \\
& \frac{\partial v}{\partial t}=u-v+D_{v} \nabla^{2} v
\end{aligned}
$$

where $u$ and $v$ correspond respectively to the concentrations of $\mathrm{HBrO}_{2}$ and the catalyst ferroin. The parameters $D_{u}=1.0$ and $D_{v}=0.6$ are the diffusion coefficients of $\mathrm{HBrO}_{2}$ and the catalyst, respectively [45]. The parameters $\epsilon=0.03$ and $q=0.002$ are chemical parameters and they are kept constant during this study. We perform studies with two different values of the parameter $f$ corresponding to rigidly rotating $(f=2.0$ ) and meandering $(f=2.5)$ scroll waves. The small parameter $\epsilon$ represents the ratio of time scales of the dynamics of the fast activator and the slow inhibitor. This parameter depends on the concentration of protons in the reactor, as $\epsilon \sim\left[\mathrm{H}^{+}\right]^{-1}[42]$.

\section{B. Extended Oregonator model}

We extended Eqs. (2) to take into account the effect of SDS in the reaction by introducing a third equation for the concentration of $\mathrm{CO}_{2}$ :

$$
\begin{aligned}
& \frac{\partial u}{\partial t}=\frac{1}{\left(\frac{\epsilon}{1+\eta c}\right)}\left[u(1-u)-f v \frac{u-q}{u+q}\right]+D_{u} \nabla^{2} u, \\
& \frac{\partial v}{\partial t}=u-v+D_{v} \nabla^{2} v, \\
& \frac{\partial c}{\partial t}=k u+G_{c} \frac{\partial c}{\partial z}+D_{c} \nabla^{2} c,
\end{aligned}
$$


where the variable $c$ corresponds to the $\mathrm{CO}_{2}$ concentration. For simplicity we assume a linear dependence of the generation of $\mathrm{CO}_{2}$ with the activator $\mathrm{HBrO}_{2}$. The diffusion coefficient of $\mathrm{CO}_{2}$ is assumed to be similar to the other components, i.e., $D_{c}=1$. The parameter $G_{c}$ accounts for the flux of $\mathrm{CO}_{2}$ due to the difference in density between the microbubbles and the rest of the reaction medium. The variable $c$ encompasses the free concentration of $\mathrm{CO}_{2}$ in the reactor and the concentration of $\mathrm{CO}_{2}$ inside the bubbles.

The transition from meandering to rigidly rotating scroll waves is achieved by changing the value of the parameter $f$. To keep the total production of $\mathrm{CO}_{2}$ constant, we modify the rate constant $k$ : for $f=2.0$ we use $k=0.003$, and for $f=2.5$ we employ $k=0.0045$. The presence of $\mathrm{CO}_{2}$ leads to an increase of the proton concentration Eq. (1), which decreases the value of the parameter $\epsilon$. To account for this change we introduce the parameter $\eta=0.2$, which estimates the changes in excitability with $\mathrm{CO}_{2}$ concentration. We consider that the new parameter $\tilde{\epsilon}$ is a function of the parameter $\epsilon$ and of the $\mathrm{CO}_{2}$ concentration:

$$
\tilde{\epsilon}=\frac{\epsilon}{1+\eta c} .
$$

To avoid numerical problems the product $\eta c$ is kept constant $\left(\eta c_{m}\right)$ for $c>c_{m}=1.5$. The value of $G_{c}$ depends on the concentration of SDS. For low concentration of SDS $\mathrm{CO}_{2}$ accumulates in bubbles which are trapped in the gel and the parameter $G_{c}$ is small. For higher concentrations of SDS, the surfactant suppresses the generation of macroscopic bubbles of $\mathrm{CO}_{2}$ and the $\mathrm{CO}_{2}$ microbubbles move upward in the gel, which is accounted for by a larger value of $G_{c}$. The accumulation of $\mathrm{CO}_{2}$ at the top of the container depends on the concentration of SDS, i.e., the parameter $G_{c}$.

\section{Boundary conditions}

We simulate a cylindrical reactor of 290-pixels height and a radius of 100 pixels inside a square domain of $220 \times 220 \times$ 290 pixel $^{3}$. On the top and bottom of the container nonflux boundary conditions are used. There is an accumulation of $\mathrm{CO}_{2}$ at the top of the container due to the nonflux boundary condition which corresponds to the experiment in a closed reactor, i.e., with a cover. When the experiment is done without cover a flux of $\mathrm{CO}_{2}$ to the exterior is observed and the nonflux condition on the top is relaxed, keeping the flux to the exterior at the top equal to the flux inside the container: $J_{z}=G_{c} \partial c / \partial z$.

To optimally reproduce the lateral boundary of the cylindrical container we employ a phase field method. We define a phase field $\phi$ as

$$
\phi(r)=\frac{1}{2}+\frac{1}{2} \tanh \left(\frac{r_{o}-r}{\xi}\right),
$$

where $r_{o}$ is the radius of the container. The phase field is $\phi=1$ inside the container and $\phi=0$ outside. We redefine the variables corresponding to the chemical concentrations by $u \rightarrow u \phi, v \rightarrow v \phi$, and $c \rightarrow c \phi$. The particular shape of the phase field variable in the boundaries given by Eq. (5) permits us to recover the nonflux boundary condition on the cylindrical boundary [46-48]. The parameter $\xi$ accounts for the size of the interface between the inside and outside of the container. It is small enough to avoid any interaction with the scroll waves and large enough to ensure numerical stability. We employ $\xi=0.6$ spatial units (s.u.), which corresponds to 2 pixels in the discretization (see Sec. IV F).

Taking into account all these considerations the equations of the model are rewritten, and they read

$$
\begin{aligned}
\frac{\partial u}{\partial t} & =\frac{1}{\left(\frac{\epsilon}{1+\eta c}\right)}\left[u(1-u)-f v \frac{u-q}{u+q}\right]+D_{u} \frac{\nabla \cdot(\phi \nabla u)}{\phi}, \\
\frac{\partial v}{\partial t} & =u-v+D_{v} \frac{\nabla \cdot(\phi \nabla v)}{\phi}, \\
\frac{\partial c}{\partial t} & =k u+G_{c} \frac{\partial c}{\partial z}+D_{c} \frac{\nabla \cdot(\phi \nabla c)}{\phi} .
\end{aligned}
$$

The variable $\phi$ is only modified to generate the initial condition (see Sec. IV D). This phase field is kept constant for the rest of the simulation. Note that inside the cylinder, which defines the container in the simulations, Eqs. (6) are reduced to the usual form of Eqs. (3).

\section{Initial condition}

We reproduce the initial condition employed in the experiments; i.e., we start the scroll wave by the partition method. To this purpose a vertical plane with nonflux boundary conditions is produced in the center of the grid of the numerical simulation by the redefinition of the parameter $\phi$ during a certain time [Fig. 6(a)]. A semicylindrical wave is initiated at the boundary of such a plane and close to the boundary of the cylindrical reactor [Fig. 6(b)]. It generates a wave which propagates toward the center of the container. The vertical plane is removed from the center [Fig. 6(c)] by fixing the phase field homogeneous inside the container, and it generates a free edge on the three-dimensional wave which produces a rotating scroll wave [Figs. 6(d)-6(f)].

\section{E. Physical lengths and times}

We choose length and time units by fixing the parameter $D_{u}=1$ and the temporal relaxation of the inhibitor. They
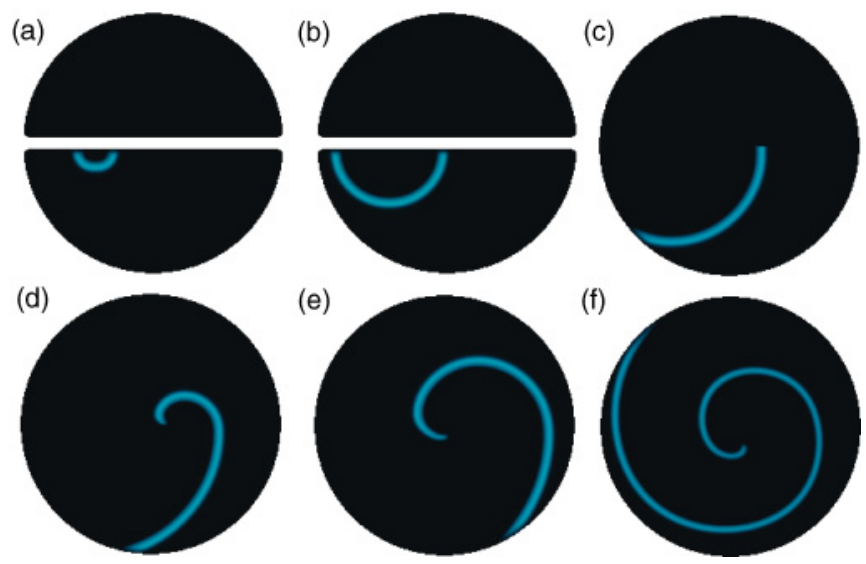

FIG. 6. (Color online) Snapshots of the activator waves in the numerical simulation corresponding to horizontal cuts of the cylindrical domain. The white line in (a) and (b) represents the partition sheet, which is removed in (c)-(f). The snapshots correspond to times $1,2,3,4,5$, and 15 t.u. The diameter of the circle is 200 s.u. 
determine a dimensionless length and a dimensionless time measured in spatial units (s.u.) and in temporal units (t.u.), respectively.

With the chosen values of the parameters of the Oregonator model and $f=2.5$ the dynamics of two-dimensional spiral waves produces outward meandering patterns with four loops [15]. The numerical dynamics under such conditions reproduce the main characteristics of the spiral waves observed in two-dimensional reactors. If we compare the characteristic size of the spiral waves and the period of rotation we obtain an equivalence of length and time between the Oregonator model and the experiments. Time and space units on the Oregonator model correspond to $1 \mathrm{t} . \mathrm{u} .=88 \mathrm{~s}$ and $1 \mathrm{s.u} .=$ $0.26 \mathrm{~mm}$. These values yield a diffusion coefficient for $\mathrm{HBrO}_{2}$ of $D_{u}=0.8 \cdot 10^{-5} \mathrm{~cm}^{2} \mathrm{~s}^{-1}$, which is comparable with the standard literature value of $D_{u}=1.5 \cdot 10^{-5} \mathrm{~cm}^{2} \mathrm{~s}^{-1}$ [45]. The disagreement between both values may refer to the inexactitude of the Oregonator model for our chemical recipe.

\section{F. Numerical methods}

We integrate the equations using the finite differences method for the spatial derivatives, which are discretized with a spatial step $\Delta x=0.3$ s.u. and using an Euler method with $\Delta t=0.001$ t.u. for the temporal integration of the equations. For the parameter values employed here the numerical method is stable.

\section{SIMULATION RESULTS}

\section{A. Different boundary conditions at the top}

We have numerically integrated the extended Oregonator model described in Eqs. (6), generating a rotating scroll wave with the initial condition described in Sec. IVD. The null production of $c(k=0)$ leads to the original Oregonator model, and scroll waves under the two sets of the parameter values ( $f=2.0$ and 2.5 ) develop the corresponding rigidly rotating and meandering motion. If the $\mathrm{CO}_{2}$ production is not zero $(k>0)$ there is a continuous generation of $c$. The presence of $c$ modifies the excitability of the system.

The advection term proportional to $G_{c}$ yields the transport of $c$ to the upper boundary of the system. The variable $c$ either accumulates with the nonflux boundary condition [Fig. 7(a)] or partially disappears through the boundary with the nonzero flux boundary condition [Fig. 7(b)]. In the first case, the accumulation at the top of the reactor generates a strong gradient in the vertical direction, while in the second case the advection produces a small gradient.

\section{B. Dynamics of rigidly rotating scroll waves}

We perform numerical simulations of a rigidly rotating scroll wave (i.e., $f=2.0$ ). The nonflux boundary condition yields the accumulation of $c$ at the top of the container. The variable $c$, which represents the $\mathrm{CO}_{2}$ concentration, enhances the excitability and therefore this $\mathrm{CO}_{2}$ accumulation produces a gradient of excitability. The reaction continuously produces $\mathrm{CO}_{2}$ and increases the excitability of the medium at the upper part of the domain. Thus, the gradient in the system grows with time. At the begin of the simulation the quantity of
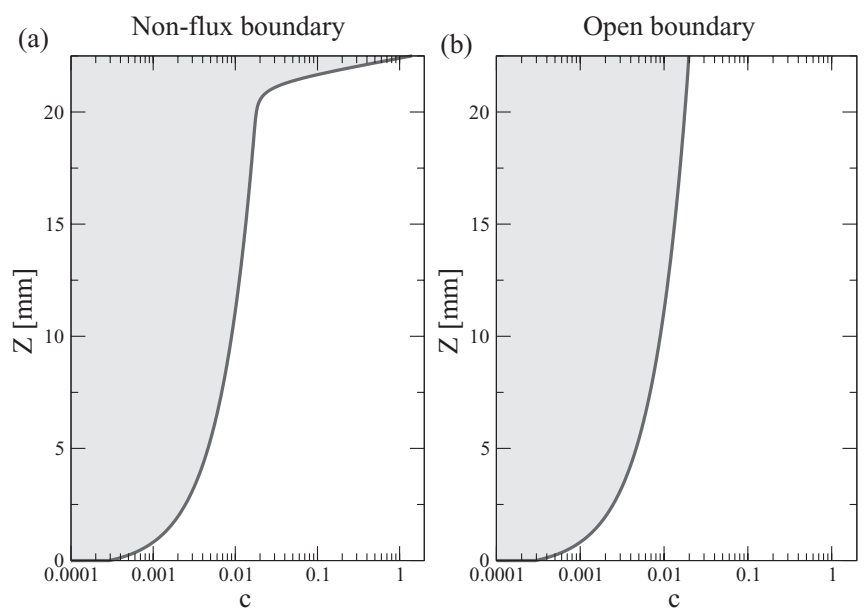

FIG. 7. Profiles of the concentration of $c$ corresponding to simulations in (a) a closed volume with a nonflux boundary condition at the top, corresponding to a covered reactor, and (b) in an open volume allowing flux through the interface at the top, corresponding to an open reactor. Note the logarithmic scale.

$\mathrm{CO}_{2}$ produced is small and the scroll wave rotates around a straight filament. After $2 \mathrm{~h}$ the gradient of $\mathrm{CO}_{2}$ becomes more evident. This gradient induces a change on the frequencies of rotation at different levels of the system, giving rise to a twist [Fig. 8(a)]. The twist begins at the top of the domain and propagates downward, thus causing the filament to adopt a slightly helicoidal shape [Fig. 8(c)]. At the end of the simulation the scroll wave is completely twisted.
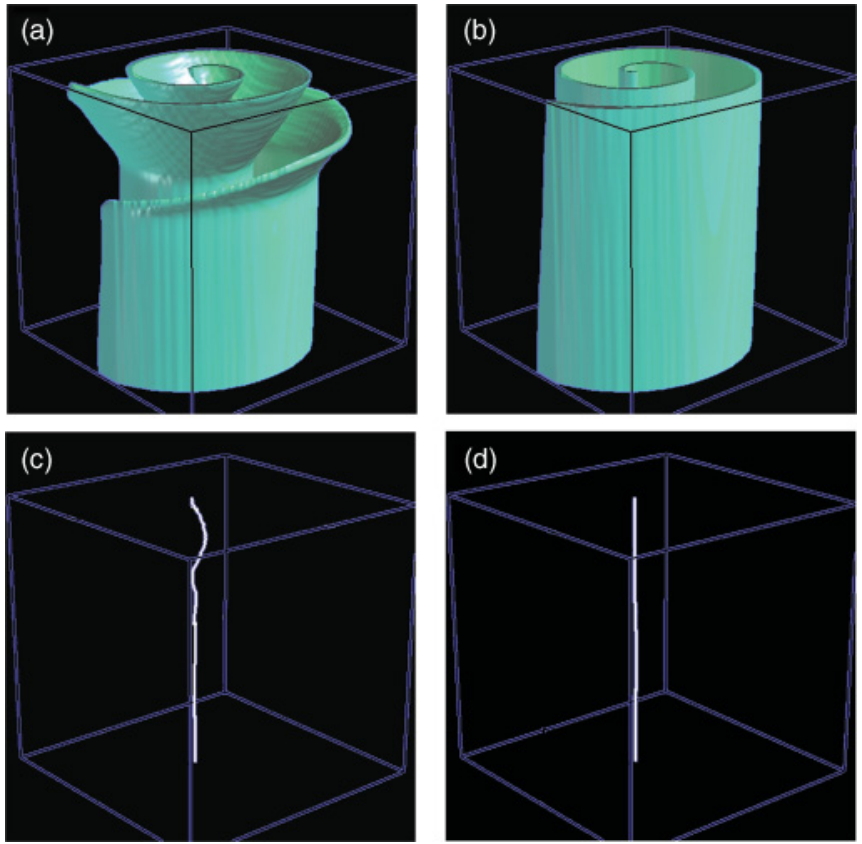

FIG. 8. (Color online) Scroll waves and filaments in simulations 235 min after initiation of the numerical simulation, in a closed volume with a nonflux boundary condition at the top (left column) and in an open volume allowing flux through the interface at the top (right column). (a) and (c) Scroll wave and filament with rigid rotation and with cover. (b) and (d) Scroll wave and filament with rigid rotation and uncovered. The size of the system is $15.6 \times 15.6 \times 22.6 \mathrm{~mm}^{3}$. 

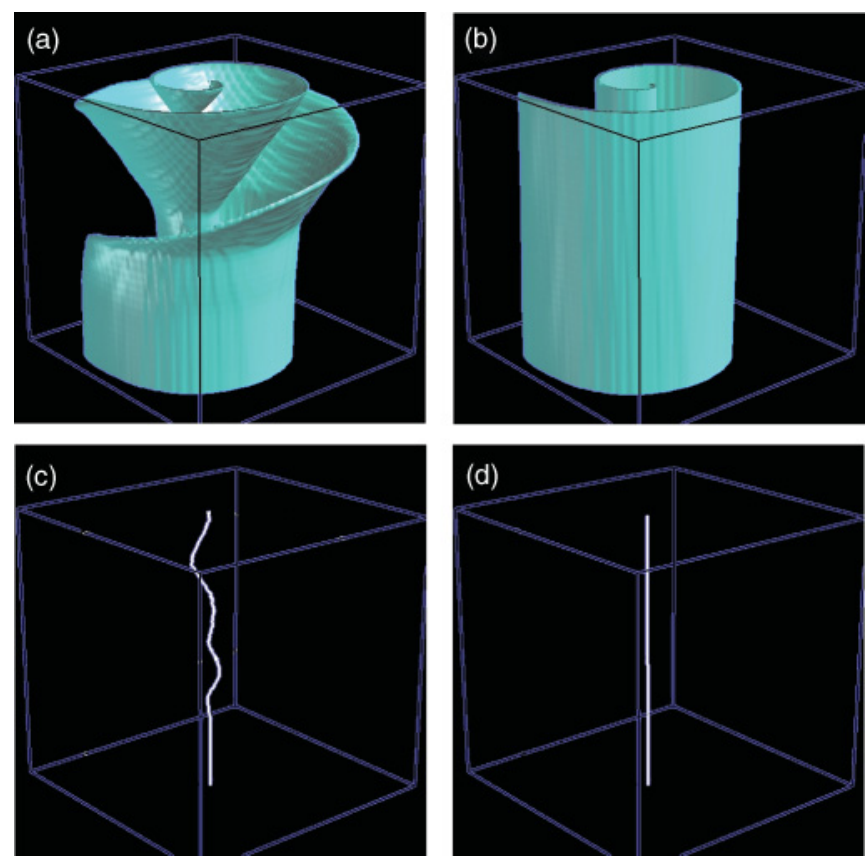

FIG. 9. (Color online) Meandering scroll waves and filaments in simulations 235 min after initiation of the numerical simulation, in a closed volume with a nonflux boundary condition at the top (left column) and in an open volume allowing flux through the interface at the top (right column). (a) and (c) Scroll wave and filament with meandering and with cover. (b) and (d) Scroll wave and filament with meandering and uncovered. The size of the system is $15.6 \times 15.6 \times$ $22.6 \mathrm{~mm}^{3}$.

If the nonflux boundary condition is broken and a certain release of the variable $c$ is allowed, $\mathrm{CO}_{2}$ no longer accumulates at the top of the reaction container. In such a case the gradient is small [Fig. 7(b)] and no twist is observed [Fig. 8(b)] while the filament remains straight [Fig. 8(d)].

\section{Dynamics of meandering scroll waves}

The results of numerical simulations of a meandering scroll wave $(f=2.5)$ are similar to the results shown in the previous section. The shape of the scroll waves and filaments under meandering conditions are displayed in Fig. 9. The accumulation of $\mathrm{CO}_{2}$, again, produces a vertical gradient which gives rise to the twist of the scroll wave [Fig. 9(a)] and to the helicoidal shape of the filament [Fig. 9(c)]. Under open boundary conditions, however, both the scroll wave and filament remain straight [Figs. 9(b) and 9(d)]. The main difference is that for a meandering scroll wave the deformation of the filament under a gradient is larger than it is for a rigidly rotating scroll [compare Figs. 8(c) and 9(c)].

\section{Effect of $\mathrm{CO}_{2}$ advection against gravity}

The production of $\mathrm{CO}_{2}$ is independent of the concentration of SDS. Thus, the value of the rate constant $k$ does not depend on the surfactant. The surfactant concentration, however, affects the size of the $\mathrm{CO}_{2}$ bubbles, which, in turn, suffer alterations in their motion upward against gravity. We modify the advection parameter $G_{c}$ in Eqs. (6) to mimic this experimental observation. This parameter con-
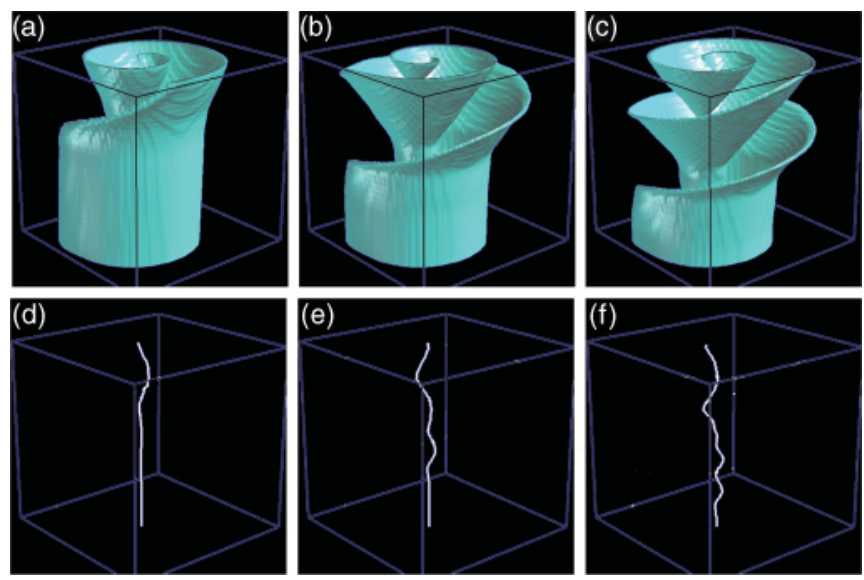

FIG. 10. (Color online) Scroll waves (a)-(c) and filaments (d)-(f) after $235 \mathrm{~min}$ of the beginning of the numerical simulation of a meandering scroll wave with a nonflux boundary condition at the top (covered) for three different values of the advection parameter: $G_{c}=0.5$ (a) and (d), $G_{c}=1$ (b) and (e), and $G_{c}=2$ (c) and (f). The size of the system is $15.6 \times 15.6 \times 22.6 \mathrm{~mm}^{3}$.

trols the rate of $\mathrm{CO}_{2}$ transport upward in the numerical simulations.

Figure 10 shows different scroll waves and their filaments resulting from numerical simulations with three different values of $G_{c}$. The magnitude of the advection parameter $G_{c}$ has an influence on the strength of the twist induced in the scroll wave. The scroll wave in Fig. 10(b) corresponds to the same conditions shown in Fig. 9(b) (i.e., using the reference value $\left.G_{c}=1\right)$. In contrast, numerical simulations with larger and lower values of $G_{c}$ give rise to scroll waves with a higher [Fig. 10(c), where $G_{c}=2$ ] and a lower twist [Fig. 10(a), where $\left.G_{c}=0.5\right]$, respectively. Note that the total amount of $\mathrm{CO}_{2}$ produced is the same in the three cases. The gradient of excitability is, however, different, producing different scroll waves. The resulting patterns successfully reproduce the experimental patterns observed in Fig. 2.

A quantitative analysis of the twist for different values of $G_{c}$ is shown in Fig. 11. The variation of the phase orientation defines the twist of the filament. Two regions are observed for each filament: above a particular height the twist is large and constant, whereas below this height the twist is small. The helicoidal shape of the filament also induces a sinusoidal

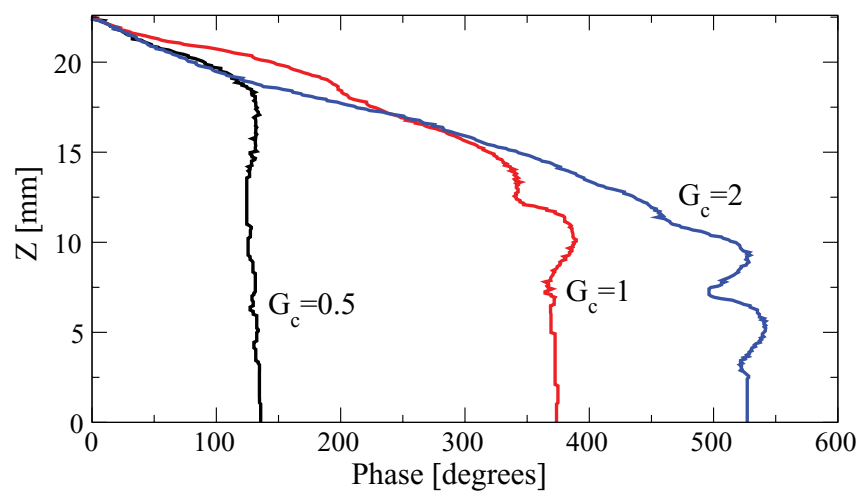

FIG. 11. (Color online) Twist of the scroll wave plotted in Fig. 10 at $225 \mathrm{~min}$ after the beginning of the numerical simulations. 
contribution to the twist where the scroll wave changes from a small to large twist. These results are in good agreement with the experimental findings (Fig. 3).

\section{DISCUSSION}

The formation of $\mathrm{CO}_{2}$ gas bubbles is an undesired feature of the classical $\mathrm{BZ}$ reaction. When performed in a gel matrix, $\mathrm{CO}_{2}$ bubbles get trapped and immobilized in the gel and very often they disrupt the spatiotemporal patterns under investigation. A popular method to diminish and even suppress the $\mathrm{CO}_{2}$ bubble formation is the addition of small concentrations of SDS to the reaction medium. In two-dimensional experiments this procedure usually leads to good results, and in three dimensions it permits a clear tomographic observation of the three-dimensional structures $[9,10]$. However, we observe that in $3 \mathrm{D}$ experiments the addition of SDS leads to the generation of a vertical gradient of excitability. The low surface tension between the reaction medium and the $\mathrm{CO}_{2}$ bubbles enhances the redissolution of $\mathrm{CO}_{2}$ into the reaction medium. This also leads to the formation of microscopic $\mathrm{CO}_{2}$ bubbles that have a smaller density than the reaction medium and, therefore, travel to the top of the reaction cylinder. Such a situation leads to the formation of a vertical $\mathrm{CO}_{2}$ gradient and, due to Eq. (1), also to a gradient in $\mathrm{H}^{+}$concentration. The magnitude of the gradient can be manipulated by changing the concentration of SDS in the reaction medium, while the existence of the gradient can be controlled by closing or opening the reaction cylinder to the atmosphere. In an open reactor, $\mathrm{CO}_{2}$ escapes to the gas phase, thus erasing the vertical $\mathrm{CO}_{2}$ gradient in the reaction medium. In the gas phase above the reactor the released $\mathrm{CO}_{2}$ forms a protective gas layer that prevents the penetration of oxygen into the reactor.

In the present article we show that SDS in the BZ reaction induces a chemical gradient in $3 \mathrm{D}$ reaction media. These gradients induce a twist at the top of the scroll waves, where both the $\mathrm{CO}_{2}$ concentration and the excitability are higher. With time the twist propagates downward along the scroll. The excitability gradients cause a dependence of the period of scroll wave rotation on the vertical direction. The scroll wave twists to compensate the changes in the period, giving rise to helicoidal or zig-zag-shaped filaments, as we have observed in both experiments and numerical simulations. Our results are consistent with previous studies on the effects of gradients in the $\mathrm{BZ}$ reaction $[9,20,23,49,50]$.

For the numerical simulations we consider the Oregonator model of the $\mathrm{BZ}$ reaction. We extend the model to take into account the effects of the $\mathrm{CO}_{2}$ concentration in the reaction. $\mathrm{CO}_{2}$ is homogeneously created by the excitation waves inside the $3 \mathrm{D}$ system with a rate that is independent of the SDS concentration. The $\mathrm{CO}_{2}$ concentration decreases the parameter $\epsilon$, increasing the excitability of the medium. An advective term is added in the equation for $\mathrm{CO}_{2}$ to take into account the formation of small bubbles and their drift in the vertical direction (i.e., against gravity). We also consider the diffusion of $\mathrm{CO}_{2}$. The numerical simulations successfully reproduce the experimental results.

The dynamics of 3D waves under gradients of excitability has been often studied in generic models of excitable media. Although linear gradients on the excitability have been occasionally employed [9,22], most studies on inhomogeneous excitability considered step gradients, which produce discontinuous excitability profiles $[9,21,22]$. Our results reproduce the main predictions of these simple models. Although the gradient produced by the $\mathrm{CO}_{2}$ is continuous, it grows exponentially at the top of the system. Therefore, the properties of the twist observed here are more similar to a step gradient. Twisted scroll waves can be also generated in the simulations without any gradient by the introduction of periodic boundary conditions [17,18,51].

Our experimental results and the modified Oregonator model provide a deeper understanding of the role of $\mathrm{CO}_{2}$ formation and accumulation in SDS-containing threedimensional BZ media. Therefore, the classical BZ reaction supplemented with sub-CMC additions of SDS can suitably be employed for the study of different problems on 3D wave propagation in excitable systems, e.g., the influence of container boundaries on a scroll wave and the interaction among several scroll waves confined in the reactor under different conditions. This opens up new avenues for the experimental and numerical studies of scroll wave dynamics using the $\mathrm{BZ}$ reaction.

\section{CONCLUSIONS}

The introduction of the surfactant SDS into the BZ reaction medium leads to the formation of a vertical gradient of excitability in the three-dimensional reaction, which in turn will result in a twist of the scroll wave. This gradient can be removed if the reaction container is not covered, thus allowing for a $\mathrm{CO}_{2}$ escape to the atmosphere. A model for the chemical reaction that takes into account the effect of SDS and of the gradient in $\mathrm{CO}_{2}$ is proposed, and results from numerical simulations are in very good agreement with the experimental results.

\section{ACKNOWLEDGMENTS}

We thank the Graduiertenförderung des Landes SachsenAnhalt for financial support for Dennis Kupitz. We acknowledge financial support by the Deutsche Forschungsgemeinschaft within the framework of SFB 910 (Control of Self-Organizing Nonlinear Systems).
[1] A. Goldbeter, Biochemical Oscillations and Cellular Rhythms: The Molecular Bases of Periodic and Chaotic Behaviour (Cambridge University Press, Cambridge, 1996).

[2] J. Keener and J. Sneyd, Mathematical Physiology (Springer, New York, 1998).
[3] A. T. Winfree, Science 266, 1003 (1994).

[4] R. H. Clayton, E. A. Zhuchkova, and A. V. Panfilov, Prog. Biophys. Mol. Biol. 90, 378 (2006).

[5] R. J. Field and M. Burger, Oscillations and Traveling Waves in Chemical Systems (Wiley, New York, 1985). 
[6] R. Kapral and K. Showalter, Chemical Waves and Patterns (Kluwer, Dordrecht, 1995).

[7] A. S. Mikhailov and K. Showalter, Phys. Rep. 425, 79 (2006).

[8] A. T. Winfree, S. Caudle, G. Chen, P. McGuire, and Z. Szilagyi, Chaos 6, 617 (1996).

[9] U. Storb, C. R. Neto, M. Bär, and S. C. Müller, PhysChemChemPhys 5, 2344 (2003).

[10] C. Luengviriya, U. Storb, G. Lindner, S. C. Müller, M. Bär, and M. J. B. Hauser, Phys. Rev. Lett. 100, 148302 (2008).

[11] T. Bánsági, K. J. Meyer, and O. Steinbock, J. Chem. Phys. 128, 094503 (2008).

[12] T. Bánsági and O. Steinbock, Chaos 18, 026102 (2008).

[13] V. N. Biktashev, A. V. Holden, and H. Zhang, Phil. Trans. R. Soc. A 347, 611 (1994).

[14] S. Alonso, F. Sagués, and A. S. Mikhailov, J. Phys. Chem. A 110, 12063 (2006).

[15] S. Alonso, F. Sagués, and A. S. Mikhailov, Science 299, 1722 (2003).

[16] H. Henry and V. Hakim, Phys. Rev. Lett. 85, 5328 (2000).

[17] H. Henry and V. Hakim, Phys. Rev. E 65, 046235 (2002).

[18] C. Henze, E. Lugosi, and A. T. Winfree, Can. J. Phys. 68, 683 (1990).

[19] A. Pertsov, R. Aliev, and V. Krinsky, Nature (London) 345, 419 (1990).

[20] S. Mironov, M. Vinson, S. Mulvey, and A. Pertsov, J. Phys. Chem. 100, 1975 (1996).

[21] A. S. Mikhailov, A. V. Panfilov, and A. N. Rudenko, Phys. Lett. A 109, 246 (1985).

[22] B. Echebarria, V. Hakim, and H. Henry, Phys. Rev. Lett. 96, 098301 (2006).

[23] T. Amemiya, P. Kettunen, S. Kádár, T. Yamaguchi, and K. Showalter, Chaos 8, 872 (1998).

[24] M. Vinson, S. Mironov, S. Mulvey, and A. Pertsov, Nature (London) 386, 477 (1997).

[25] C. Luengviriya, S. C. Müller, and M. J. B. Hauser, Phys. Rev. E 77, 015201(R) (2008).

[26] C. Luengviriya and M. J. B. Hauser, Phys. Rev. E 77, 056214 (2008).

[27] K. Kurin-Csörgei, I. Szalai, and E. Körös, React. Kinet. Catal. Lett. 54, 217 (1995).

[28] K. Kurin-Csörgei, A. M. Zhabotinsky, M. Orbán, and I. R. Epstein, J. Phys. Chem. 100, 5393 (1996).
[29] A. Lázár, Z. Noszticzius, H.-D. Försterling, and Z. NagyUngvárai, Physica D 84, 112 (1995).

[30] K. Agladze, V. Voigner, E. Hamm, F. Plaza, and V. Krinsky, J. Phys. Chem. 100, 18764 (1996).

[31] A. Belmonte, J.-M. Fleselles, and Q. Ouyang, Europhys. Lett. 35, 665 (1996).

[32] C. Luengviriya, U. Storb, M. J. B. Hauser, and S. C. Müller, PhysChemChemPhys 8, 1425 (2006).

[33] J. S. Park and K. J. Lee, Phys. Rev. E 73, 066219 (2006).

[34] M. Maritato, J. Nikles, L. S. Romsted, and M. Tramontin, J. Phys. Chem. 89, 1341 (1985).

[35] F. P. Cavassino, R. Cervelatti, R. Lombardo, and M. L. Turco Liveri, J. Phys. Chem. B 103, 4285 (1999).

[36] A. Paul, J. Phys. Chem. B 109, 9639 (2005).

[37] L. Sciascia, R. Lombardo, and M. L. Turco Liveri, J. Phys. Chem. B 111, 1354 (2007).

[38] S. Ristori, F. Rossi, G. Biosa, N. Marchettini, M. Rustici, and E. Tiezzi, Chem. Phys. Lett. 436, 175 (2007).

[39] F. Rossi, R. Lombardo, L. Sciascia, C. Sbrizolo, and M. L. Turco Liveri, J. Phys. Chem. B 112, 7244 (2008).

[40] G. Biosa, S. Rossi, O. Spalla, M. Rustici, and M. J. B. Hauser, J. Phys. Chem. A 115, 3227 (2011).

[41] D. Stock and S. C. Müller, Physica D 96, 396 (1996).

[42] R. J. Field, Scholarpedia 2, 1386 (2007).

[43] A. Rusakov, A. B. Medvinsky, and A. V. Panfilov, Phys. Rev. E 72, 022902 (2005).

[44] J. J. Tyson and P. C. Fife, J. Chem. Phys. 73, 2224 (1980).

[45] W. Jahnke and A. T. Winfree, Int. J. Bif. Chaos 1, 445 (1991).

[46] J. Kockelkoren, H. Levine, and W.-J. Rappel, Phys. Rev. E 68, 037702 (2003).

[47] H. Levine and W.-J. Rappel, Phys. Rev. E 72, 061912 (2005).

[48] F. H. Fenton, E. M. Cherry, A. Karma, and W.-J. Rappel, Chaos 15, 013502 (2005).

[49] C. Zhang, H. Zhang, Q. Ouyang, B. Hu, and G. H. Gunaratne, Phys. Rev. E 68, 036202 (2003).

[50] C. Qiao, Y. Wu, X. C. Lu, C. Y. Wang, Q. Ouyang, and H. Wang, Chaos 18, 026109 (2008).

[51] D. Margerit and D. Barkley, Phys. Rev. Lett. 86, 175 (2001). 